\title{
Whole-brain 3D MR fingerprinting brain imaging: clinical validation and feasibility to patients with meningioma
}

\author{
Thomaz R. Mostardeiro ${ }^{1}$ (I) $\cdot$ Ananya Panda ${ }^{1} \cdot$ Robert J. Witte $^{1} \cdot$ Norbert G. Campeau $^{1} \cdot$ Kiaran P. McGee $^{1} \cdot$ Yi Sui $^{1}$. \\ Aiming $\mathrm{Lu}^{1}$
}

Received: 18 December 2020 / Revised: 29 March 2021 / Accepted: 19 April 2021 / Published online: 4 May 2021

(c) The Author(s) 2021

\begin{abstract}
Purpose MR fingerprinting (MRF) is a MR technique that allows assessment of tissue relaxation times. The purpose of this study is to evaluate the clinical application of this technique in patients with meningioma.

Materials and methods A whole-brain 3D isotropic $1 \mathrm{~mm}^{3}$ acquisition under a 3.0T field strength was used to obtain MRF $\mathrm{T}_{1}$ and $\mathrm{T}_{2}$-based relaxometry values in 4:38 $\mathrm{s}$. The accuracy of values was quantified by scanning a quantitative MR relaxometry phantom. In vivo evaluation was performed by applying the sequence to 20 subjects with 25 meningiomas. Regions of interest included the meningioma, caudate head, centrum semiovale, contralateral white matter and thalamus. For both phantom and subjects, mean values of both $\mathrm{T}_{1}$ and $\mathrm{T}_{2}$ estimates were obtained. Statistical significance of differences in mean values between the meningioma and other brain structures was tested using a Friedman's ANOVA test.

Results MR fingerprinting phantom data demonstrated a linear relationship between measured and reference relaxometry estimates for both $\mathrm{T}_{1}\left(r^{2}=0.99\right)$ and $\mathrm{T}_{2}\left(r^{2}=0.97\right)$. MRF $\mathrm{T}_{1}$ relaxation times were longer in meningioma (mean $\pm \mathrm{SD}$ $1429 \pm 202 \mathrm{~ms}$ ) compared to thalamus (mean \pm SD $1054 \pm 58 \mathrm{~ms} ; p=0.004)$, centrum semiovale (mean \pm SD $825 \pm 42 \mathrm{~ms}$; $p<0.001)$ and contralateral white matter (mean $\pm \mathrm{SD} 799 \pm 40 \mathrm{~ms} ; p<0.001)$. MRF $\mathrm{T}_{2}$ relaxation times were longer for meningioma (mean $\pm \mathrm{SD} 69 \pm 27 \mathrm{~ms}$ ) as compared to thalamus (mean $\pm \mathrm{SD} 27 \pm 3 \mathrm{~ms} ; p<0.001$ ), caudate head (mean $\pm \mathrm{SD}$ $39 \pm 5 \mathrm{~ms} ; p<0.001)$ and contralateral white matter (mean \pm SD $35 \pm 4 \mathrm{~ms} ; p<0.001$ )

Conclusions Phantom measurements indicate that the proposed 3D-MRF sequence relaxometry estimations are valid and reproducible. For in vivo, entire brain coverage was obtained in clinically feasible time and allows quantitative assessment of meningioma in clinical practice.
\end{abstract}

Keywords Whole-brain MR fingerprinting $\cdot$ Meningioma $\cdot$ Relaxometry $\cdot$ 3D isotropic

\section{Introduction}

Magnetic resonance fingerprinting (MRF) is a novel MR acquisition method for quantitative assessment of tissue magnetic properties such as $\mathrm{T}_{1}, \mathrm{~T}_{2}$ and proton density [1-3]. Introduced in 2013, MRF involves acquiring either a two or three-dimensional dataset typically using non-Cartesian $\mathrm{k}$-space encoding sampling scheme such as a spiral trajectory $[2,4,5]$. Unlike conventional acquisitions which commonly require establishment of a steady state of the magnetization before spatial encoding, a MRF pulse sequence modifies

Thomaz R. Mostardeiro

thomaz.r.mostardeiro@gmail.com

1 Department of Radiology, Mayo Clinic, Rochester, MN, USA acquisition parameters, including the radiofrequency flip angle, the pulse repetition rate (TR) and echo time (TE), over a time interval while continuously acquiring data [6-8]. For a given voxel in the reconstructed MR images and given that the acquisition parameters are known, a simulated or equivalent MR signal time course can be generated for a given set of relaxometry values [9-11]. For each voxel, the signal evolution obtained is compared with a collection of simulated signal evolutions (or fingerprints). The best match for the voxel fingerprint is selected from this collection (the dictionary) through a pattern matching process. Processing of all voxels in this manner results in the generation of quantitative spatial relaxometry maps $[10,11]$, thereby providing a method to assess the underlying magnetic properties of the tissue in both normal and disease states [11-14]. 
Phantom experiments have validated the accuracy and precision of MRF relaxometry estimates [8, 11, 14-16using a quantitative relaxometry phantom developed jointly by the National Institute of Science and Technology (NIST) and the International Society of Magnetic Resonance in Medicine (ISMRM) [15] as well as agarose gel [12, 17, 18] and T1MES [19] phantoms. Also, under in-vivo neuroimaging, MRF relaxometry has been demonstrated to be repeatable and reproducible across multiple scanners [20] and field strengths [21]. Clinically it has also been demonstrated that 2D-MRF-based relaxometry can identify abnormalities that are poorly visualized using conventional MR imaging in patients with epilepsy [7, 22]. In addition, slice selective 2D-MRF has been proposed for differentiating intra-parenchymal brain tumors, such as: high-grade gliomas [23, 24] (World Health Organization grades III and IV), low-grade gliomas [23, 24] (World Health Organization grades I and II) and metastases [23]. Given the mentioned investigations in neuroimaging [22-24] studied only selected 2D-MRF slices of the brain, 3D-MRF has been proposed [5, 25-27] to allow a fast whole-brain coverage in a Radiation Oncology setting [25] and in patients with Parkinson Disease [28]. These studies [25, 28] demonstrated that a fast entire brain coverage was feasible with high resolution, pointing a major advantage of a 3D-MRF acquisition for clinical applications.

Meningioma are classified by the World Health Organization into three grades: grade I (benign), grade II (atypical meningioma), and grade III (anaplastic or malignant meningioma) [29], accounting for $13-26 \%$ of intracranial tumors with the vast majority $(85 \%)$ being benign $[29,30]$. Quantitative imaging techniques have been proposed for tissue characterization in meningioma, such as ADC values [31-33] and MR elastrography [34]. This pilot investigation has two goals. First, it aims to further validate the relaxometry estimations from a whole-brain 3D-MRF [9] acquisition through a NIST/ISMRM phantom experiment. Second, it aims to evaluate the clinical feasibility of this sequence into a clinical cenario by providing further insight for relaxometry properties in charaterization of meningiomas and selected brain structures.

\section{Methods}

\section{Image acquisition and reconstruction}

All exams were acquired on two 3T clinical MR scanners (Discovery MR750 and Discovery MR750W, GE Healthcare, Waukesha, WI) using an eight channel receive-only RF head coil. MRF data acquisition was performed using a three-dimensional steady state free precession sequence with a novel multi-axis spiral trajectory and slab excitation [9]. Adiabatic inversion pulses were used before each acquisition. A ramp flip angle schedule was used ranging from $0.778^{\circ}$ to $70^{\circ}$. The sequence details can be found in [9]. A volumetric k-space data set consisting of $256 \times 256 \times 256$ samples and a FOV of $25.6 \times 25.6 \times 25.6 \mathrm{~cm}^{3}$ resulted in reconstructed relaxometry maps with $1 \mathrm{~mm}$ isotropic resolution. The total acquisition time for the whole brain was 4:38 (minutes: seconds). The $\mathrm{T}_{1}$ for the dictionary ranged from 10 to $3000 \mathrm{~ms}$ and $\mathrm{T}_{2}$ from $10 \mathrm{~ms}$ up to $2000 \mathrm{~ms}$. Each $\mathrm{T}_{1}$ and $\mathrm{T}_{2}$ dictionary step range follow the exponential nature of $\mathrm{T}_{1}$ and $\mathrm{T}_{2}$ relaxation curves. For example, 10:5:100 means step size 5 range from 10 to $100 \mathrm{~ms}$. As such, a few examples for $\mathrm{T}_{1}$ curve steps are 10:5:100, 110:10:1000, 1050:50:2000, 2100:100:3000 and for $\mathrm{T}_{2}=10: 1: 100$, 105:5:500, 525:25:1000, 1100:100:2000. Fingerprint reconstruction and dictionary matching were performed offline in Matlab (Mathworks, Natick, Massachusetts) on a Linux workstation equipped with two 8-core Intel Xeon Gold 6244 central processing unit @ $3.60 \mathrm{GHz}, 376$ GB system memory, and a NVIDIA Tesla V100 graphical processing unit. The reconstruction pipeline has been described elsewhere [35]. Briefly, the undersampled data were anti-aliased with a k-space-weighted view-sharing algorithm and [36, 37] then the view-shared data were compressed with singular value decomposition algorithm and finally the first 15 singular value decompensation coefficients of the temporal signals were kept for parametric maps reconstruction. GPU gridding and fast Fourier transform were performed on the compressed non-Cartesian k-space data. $\mathrm{T}_{1}, \mathrm{~T}_{2}$ and proton density maps were computed via dictionary matching on the general processing unit and interpolated to $512 \times 512 \times 512$ image matrix for display. The computation time was approximately $10 \mathrm{~min}$ for each dataset.

\section{Phantom validation}

To evaluate the accuracy and reproducibility of the MRFbased relaxometry maps, a quantitative MR relaxometry phantom developed jointly by the NIST and ISMRM [38] was scanned 7 times over the course of $30 \mathrm{~min}$. This phantom has compartments containing solutions with a wide range of $T_{1}$ and $T_{2}$ values [15]. MRF-based $T_{1}$ and $T_{2}$ values were compared with the calibrated nominal values provided with the phantom.

\section{Patient studies}

An institutional review board approved study was used to acquire MRF data in patients scheduled for a diagnostic brain MR examination. The MRF sequence was added to the clinical protocol and acquired prior to the administration of a gadolinium-based contrast agent to assess the native relaxometry of the in-vivo brain. 
Only patients with biopsy-proven or imaging diagnosis of meningioma identified on prior diagnostic MRI examinations were included. Radiation induced meningioma (RIM) were included but those that were treated with gamma knife stereotactic radiosurgery were excluded. Subject ages ranged from 18- to 76-years old. A total of 22 patients were recruited with two being excluded from the statistical analysis. The first patient was excluded due to the small size of the mass and the effect of partial volume averaging (Fig. 1) on relaxometry values while the second was excluded due to concerns about being a potential extradural metastasis instead of meningioma on expert neuroradiology re-review. The final population comprised twenty patients; four patients had multiple tumors thus totaling 25 meningiomas. Seventeen tumors were non-treated meningiomas (NTM), four partially resected meningiomas (PRM) and four prior brainradiation-induced meningiomas (RIM). Pathology was available for nine meningiomas ( $3=$ Grade I; $6=$ Grade II) in different groups: two RIM, three NTM and all four PRM. For NTM with biopsy results available, the MRF scan was done before biopsy to avoid tract changes. PRM were included only if the residual tumor was clearly defined without any described operative change.

\section{MRF image analysis}

To estimate in-vivo brain relaxometry values, regions of interest (ROI) were drawn directly over T1 MRF relaxometry maps using the ITK-SNAP software Version 3.8 [39]. ROIs were then copied to MRF $T_{2}$ maps. Clinical pre- and post-contrast MRI images were used as reference for all 15 study patients. ROIs were drawn to include the meningioma as well as normal structures that included the centrum semiovale (CS), thalamus, contralateral white matter $(\mathrm{CWM})$ and caudate head $(\mathrm{CH})$. The rationale in selecting normal brain structures that are both on deep gray matter and white matter is to compare how the MRF numbers we described on those structures are in accordance to more established literature descriptions on relaxometry in these same structures. All ROI were reviewed by board-certified neuroradiologists prior to finalization. Figure 2 illustrates ROIs used to quantify these regions. If the meningioma crossed the midline, then the CWM ROI in the hemisphere opposite to the epicenter of the lesion was chosen. In those patients who had more than one meningioma, the location of the CWM ROI was chosen in the hemisphere opposite the largest lesion.

\section{Statistical analysis}

To assess the precision and accuracy of MRF-based phantom relaxometry values, a linear regression analysis (Microsoft Excel 2010, Microsoft Corporation, Redmond, WA) was performed on the MRF phantom data. Repeatability was calculated by measuring the standard deviation of the phantom values over the seven repeat acquisitions obtained over the course of the 30-min imaging session.

The means of the MRF-based $\mathrm{T}_{1}$ and $\mathrm{T}_{2}$ values for meningioma and normal brain structures were compared using a nonparametric Friedman's Test ANOVA (OriginPro 2020b, Northampton, MA) that included a post-hoc Dunn's analysis to determine statistical significance between ROI. In patients with multiple tumors, each meningioma was paired multiple times with normal brain structures. A $p$ value $<0.05$ was used for statistical significance.
Fig. 1 Anatomic and MRF data of subject excluded due to partial volume effects. a MRF $\mathrm{T}_{1}$-relaxometry map shows a small dural-based right parietal meningioma overlying the right operculum with the lowest $\mathrm{T}_{1}$ value $(793 \mathrm{~ms})$. This was also the smallest lesion $(0.9 \mathrm{~cm})$ in this study. b Axial spin echo post-gadolinium $\mathrm{T}_{1} \mathrm{WI}$ shows typical homogeneous enhancement and extradural location of meningioma
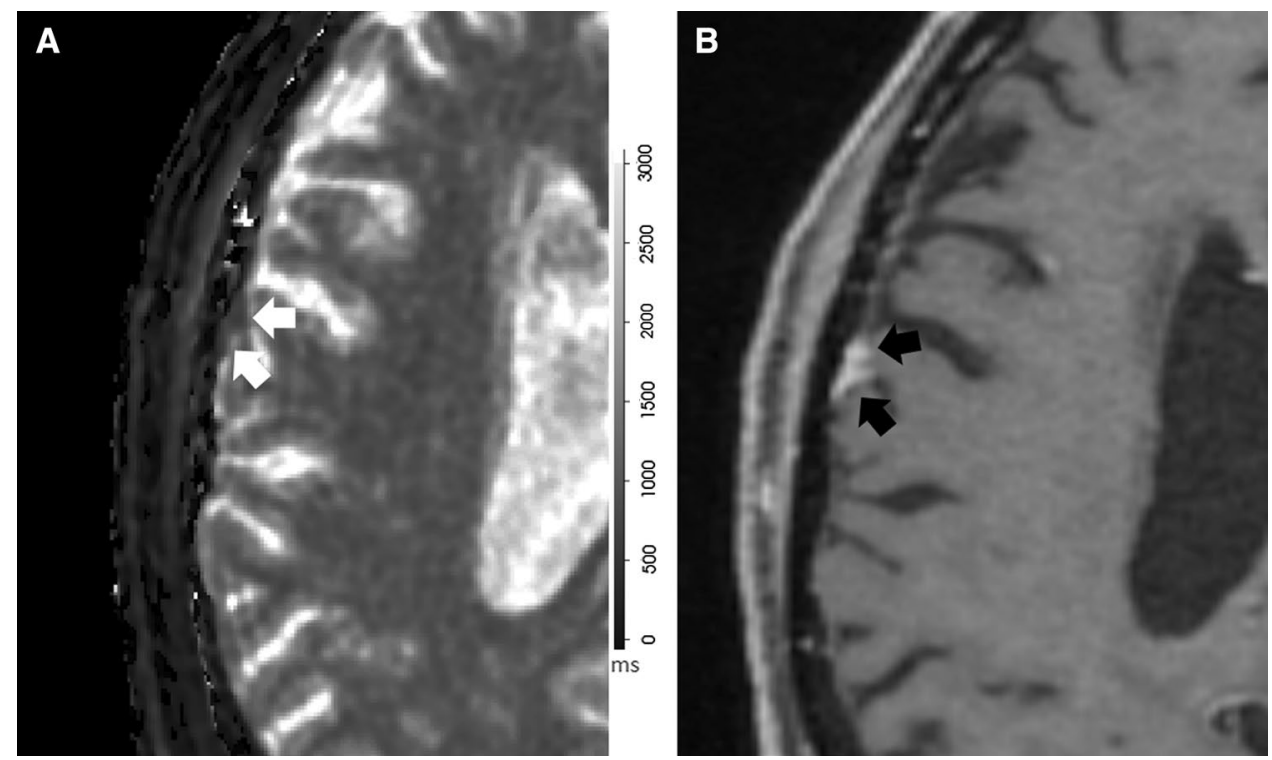
Fig. 2 MRF-derived $T_{1}$ relaxation maps from the same subject. a Depicts ROI drawings in the (1) meningioma and (2) contralateral white matter (CWM). b Illustrates the ROIs used to encompass the caudate head $(\mathrm{CH})(3)$ and thalamus (4). The grayscale is calibrated in milliseconds (ms), shown to the right of the respective relaxation maps
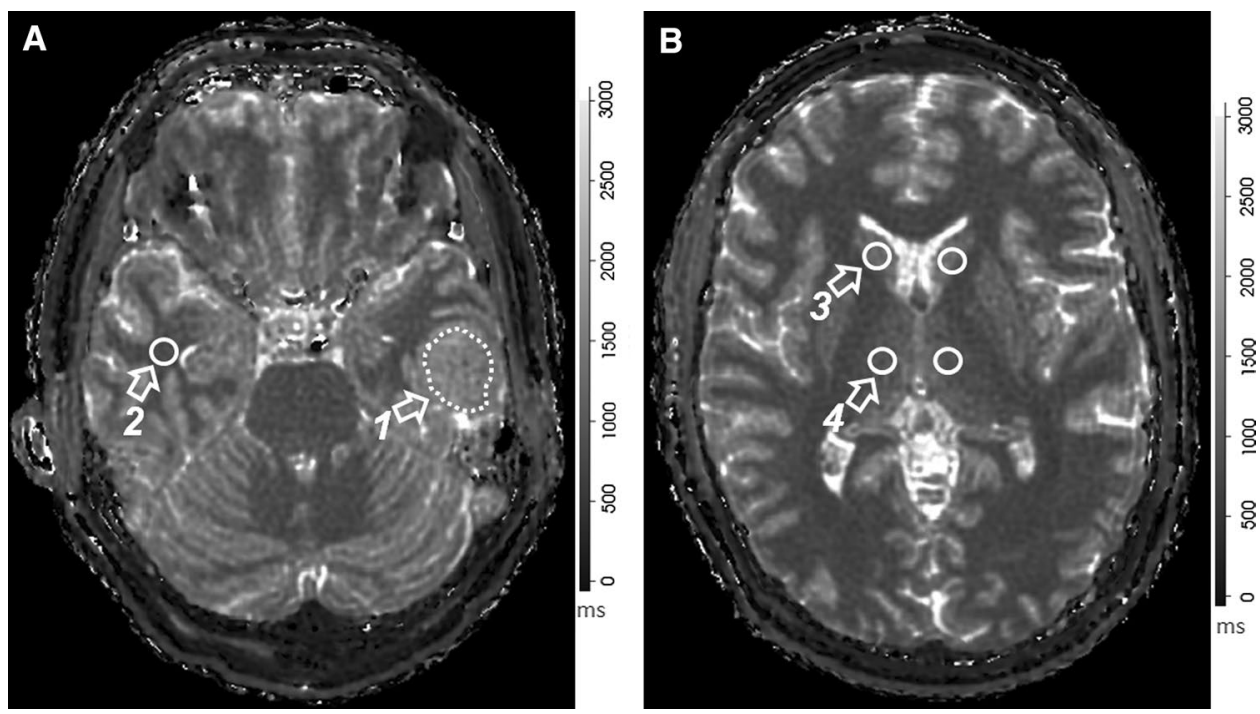

\section{Results}

\section{Phantom}

MRF-based $T_{1}$ and $T_{2}$ relaxation times of the NIST phantom in the $\mathrm{T}_{1}$ array layer are shown in Fig. 3. Figure 3a, $\mathrm{b}$ depict $\mathrm{T}_{1}$ and $\mathrm{T}_{2}$ relaxation maps through the center of the phantom identifying the 14 inserts used to estimate the respective relaxometry values. The standard deviation along the mean for seven MRF measurements as a function of $T_{1}$ and $\mathrm{T}_{2}$ relaxation times was calculated for each vial and ranged from 0.4 to $6.8 \mathrm{~ms}$ and 0.4 to $12.1 \mathrm{~ms}$ for $\mathrm{T}_{1}$ and $\mathrm{T}_{2}$, respectively. Figures 3(C) show the mean measured versus reference relaxometry values for $\mathrm{T}_{1}$ and $\mathrm{T}_{2}$ up to $1600 \mathrm{~ms}$ (D) and $200 \mathrm{~ms}$ (E). While both $\mathrm{T}_{1}$ and $\mathrm{T}_{2}$ MRF-based relaxometry were highly linear correlated $\left(\mathrm{T}_{1}: r^{2}=0.99\right.$; $\mathrm{T}_{2}: r^{2}=0.9915-200 \mathrm{~ms} ; \mathrm{T}_{2}: r^{2}=0.9715 \mathrm{~ms}-1600 \mathrm{~ms}$ ), there was a higher agreement for $\mathrm{T}_{1}$ compared to $\mathrm{T}_{2}$ values and is reflected in the mean absolute percentage error for MRF-based relaxometry which was $11 \%$ for $\mathrm{T}_{1}$ and $27 \%$ for $\mathrm{T}_{2}$. The mean absolute percentage error was lower (3\% for $\mathrm{T}_{1}$ and $14 \%$ for $\mathrm{T}_{2}$ ) for the clinically relevant relaxation times from all the regions analyzed in this study $\left(\mathrm{T}_{1}\right.$ range 500-2000 $\mathrm{ms}$ and $\mathrm{T}_{2}$ range $15-200 \mathrm{~ms}$ ).

\section{In vivo}

Eleven of the 20 patients (mean age of $59 \pm 13$ years $($ mean $\pm S D)$ were female. The median time between initial diagnosis to MRF imaging was 13 months (0-198 months). For patients with multiple meningiomas $(n=4)$, a single patient had three lesions (2 RIM, 1 PRM) while the remaining three had two lesions each (two patients had two NTM; another patient; two RIM). Grade I meningioma $(n=6)$ had a mean $\mathrm{T}_{1} \pm \mathrm{SD}$ and $\mathrm{T}_{2} \pm \mathrm{SD}$ of $1436 \pm 72 \mathrm{~ms}$ and $65 \pm 34 \mathrm{~ms}$, respectively, while Grade II $(n=3)$ had a mean $\mathrm{T}_{1}$ and $\mathrm{T}_{2}$ of $1599 \pm 388$ and $90 \mathrm{~ms} \pm 53$. Representative MRF maps obtained from one patient are shown in Fig. 4. Figure 5 shows the box and whisker plots for differences in $T_{1}$ and $T_{2}$ relaxation times between meningioma and normal structures.

Table 1 lists the statistical analysis of MRF-based relaxometry data for all patients. Mean $\mathrm{T}_{1}$ relaxation times were significantly longer for meningioma when compared to the thalamus $(p=0.001)$, CS $(p<0.001)$ and CWM $(p<0.001)$. Deep gray matter showed longer $\mathrm{T}_{1}$ relaxation compared to white matter, as represented by the $\mathrm{CH}$ versus the $\mathrm{CS}$ $(p<0.001)$ and CWM $(p<0.001)$ and the thalamus compared to CWM $(p<0.001)$. All other statistical comparisons between anatomical structures were not significant. Mean $\mathrm{T}_{2}$ relaxation of meningioma times were longer than the thalamus ( $p<0.001), \mathrm{CH}(p<0.001), \mathrm{CWM}(p<0.001) . \mathrm{T}_{2}$ relaxation times of the CS was significantly longer than the thalamus $(p<0.001)$. All other pair-wise comparisons for $\mathrm{T}_{2}$ were not statistically significant.

\section{Discussion}

MRF allows accurate and time-efficient quantitative mapping of MR tissue relaxometry values across various organ systems [11, 14]. In neuroimaging, MRF has been used for evaluation of intracranial tumors [23, 24, 40] and epilepsy $[7,22,41]$. To our knowledge, this is the first study to report on the use of this technique in a whole-brain 3D scheme covering the entire brain in patients with meningioma under routine clinical imaging conditions. Using the interleaved MRF spiral acquisition, isotropic coverage of the whole brain at a resolution of $1 \mathrm{~mm}^{3}$ per voxel was achieved in less than 5 min of acquisition time (4:38 min: seconds). Validation was obtained using a standardized phantom where 

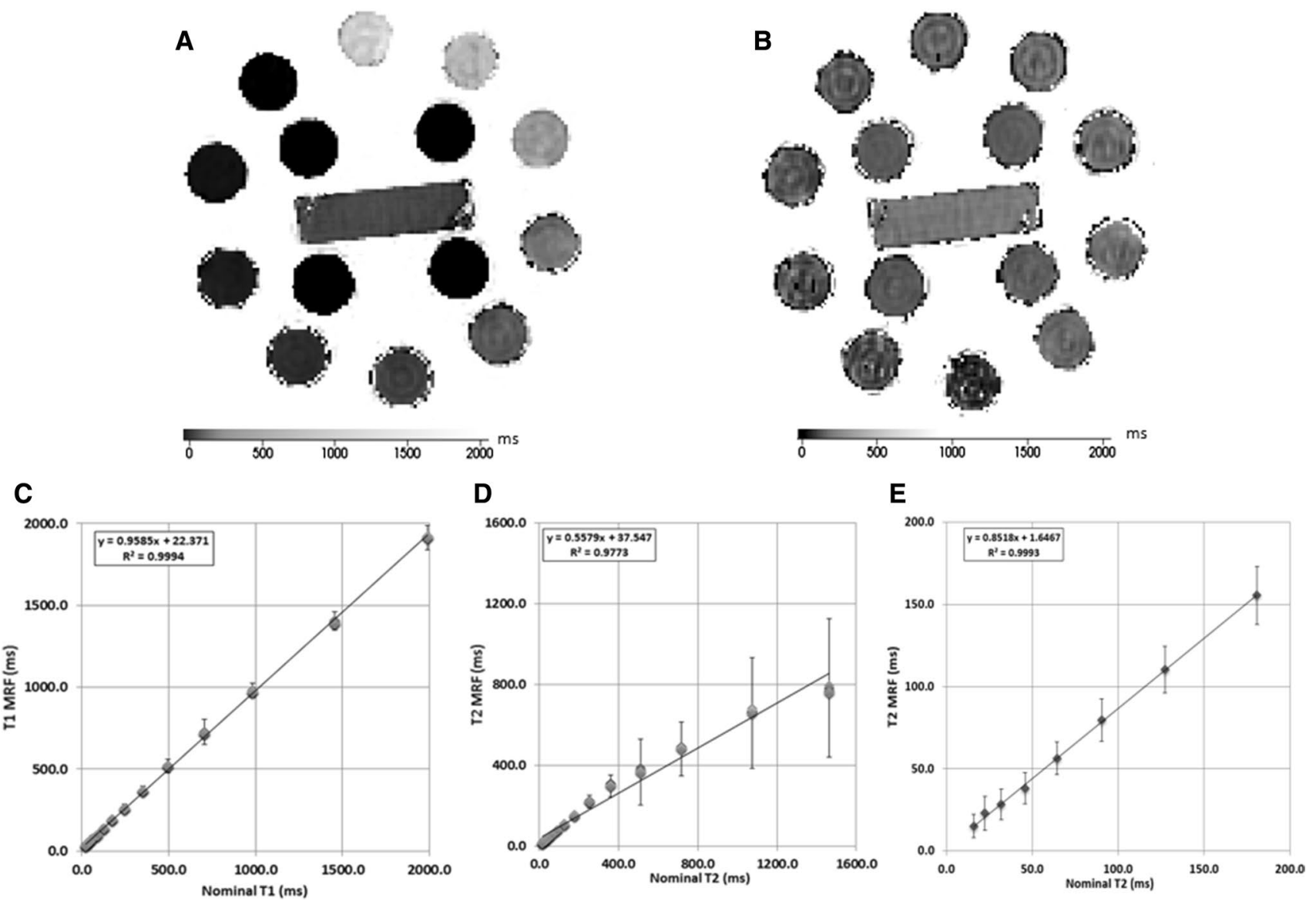

Fig. 3 Phantom validation. a, b $\mathrm{T}_{1}$ relaxation maps $\mathrm{A}$ and $\mathrm{T}_{2}$ relaxation maps $B$ for the phantom's inserts from one acquisition in the $T_{1}$ array layer. c, $\mathbf{d}$ Scatter plots of MRF-based relaxation times ( $y$-axis) versus nominal relaxation times ( $x$-axis) for $\mathrm{T}_{1} \mathrm{C}$ and in $\mathrm{T}_{2}$ up to $1600 \mathrm{~ms} \mathrm{D}$ and $200 \mathrm{~ms}$ E show linear strong correlation for MRF- based relaxation times compared to nominal relaxation times for both $\mathrm{T} 1$ and $\mathrm{T} 2$, with underestimation for both properties at higher ranges of $T_{1}$ and $T_{2}$. The error bars represent the mean standard deviation within the regions of interest within each phantom's insert three-dimensional MRF-based relaxometry values were linearly correlated with their stated reference values $\left(r^{2}=0.99\right.$ for $\mathrm{T}_{1}$ and $\mathrm{T}_{2}$ ) over the range of clinically relevant $\mathrm{T}_{1}$ and $\mathrm{T}_{2}$ relaxometry values.

In the phantom data presented perfect agreement between the calculated and stated relaxometry values was not achieved across the range of $T_{1}$ and $T_{2}$ values tested. This difference was more evident in $\mathrm{T}_{2}$ compared to $\mathrm{T}_{1}$ estimates and most prominent at the longest $T_{2}$ value $(1500 \mathrm{~ms})$ (Fig. 3c-e). Within the context of this study, the impact of these variations is minimal as the in-vivo brain tissue estimates are characterized by $T_{2}$ values of less than $200 \mathrm{~ms}$ at 3T [42] and, therefore, expected to be accurately determined using the proposed MRF technique. Furthermore, the relaxometry range at $3 \mathrm{~T}$ described for peritumoral white matter (including edema), glioblastoma multiforme, low-grade gliomas and metastatic disease is bellow $200 \mathrm{~ms}$ [23, 24]. While similar conclusions cannot be made with regards to MRF-based estimates of CSF, which is known to have a $\mathrm{T}_{2}$ values of the order of 1000-2000 ms at 3T [43], estimates of CSF were not made and therefore do not impact the results of this study.

There are a few potential reasons for the deviation between the measured and nominal relaxation times for MRF-based $T_{2}$ values. First, the pulse sequence used employs a spoiled gradient echo acquisition scheme resulting in a highly $\mathrm{T}_{1}$-weighted MR signal $[2,9]$. Second, the sequence does not employ any additional $\mathrm{T}_{2}$ preparation pulses to provide greater $T_{2}$ weighting thereby being less sensitive to tissues with long $\mathrm{T}_{2}$ relaxation times. Third, the sequence does not account for effects such as magnetization transfer, diffusion and field inhomogeneities which can affect relaxometry estimates $[9,44]$.

MRF $T_{1}$ and $T_{2}$ relaxation values of the selected normal brain structures in this study are in agreement with previously published results derived from methods using a 2D acquisition [20, 42]. Badve et al. [42] investigated MRFbased relaxation in white matter structures from selected 4-5 

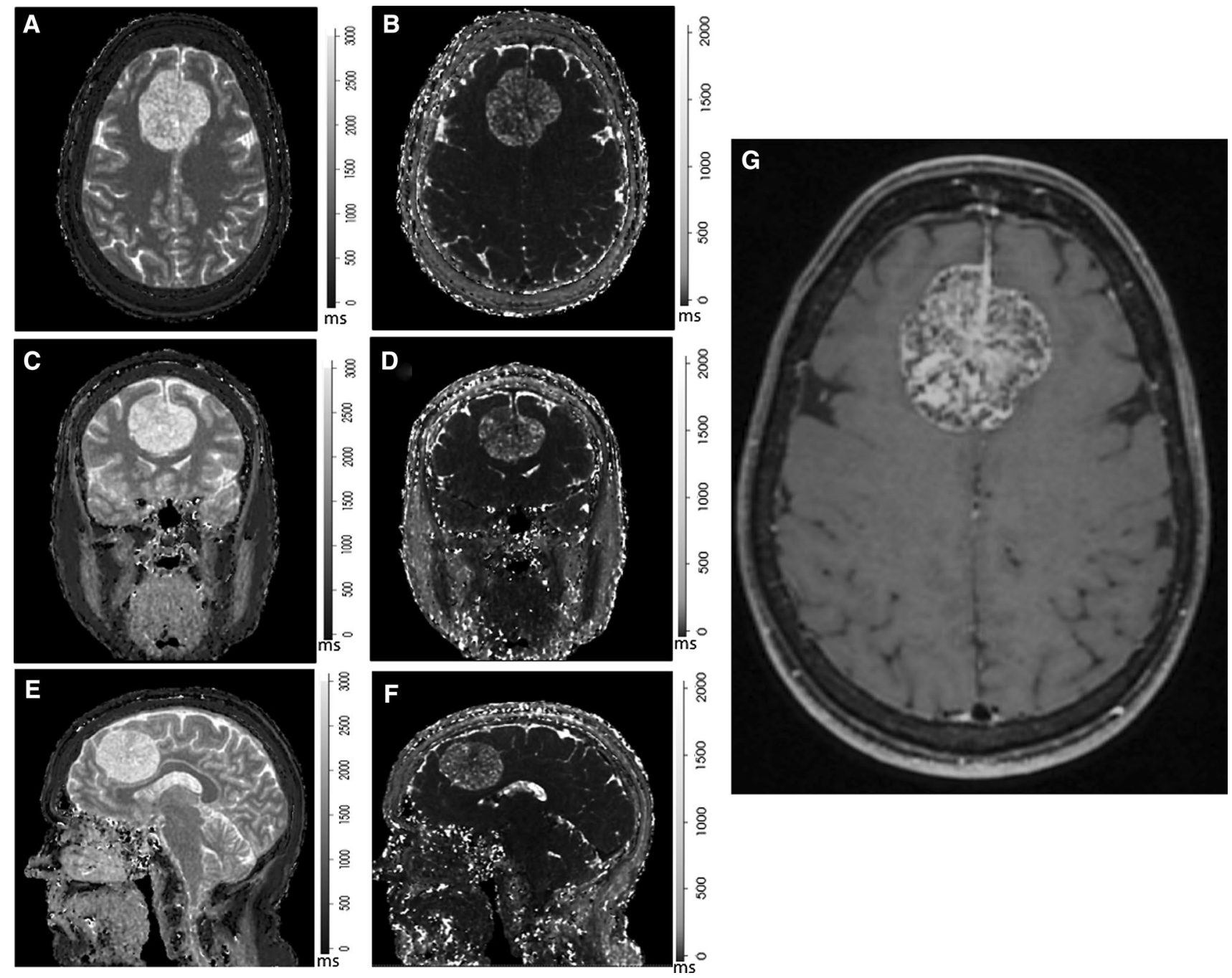

Fig. 4 MRF relaxometry maps on axial a, b, coronal $\mathbf{c}$, d, sagittal $\mathbf{e}, \mathbf{f}$ and $\mathrm{T}_{1} \mathrm{WI}$ post gadolinium $\mathbf{g}$ in a pathology proven atypical meningioma (Grade II). MRF-derived $\mathrm{T}_{1}$-relaxometry (a, c, e) and $\mathrm{T}_{2}$-relaxometry $(\mathbf{b}, \mathbf{d}, \mathbf{f})$ maps show large inter-hemispheric meningi-

brain slices, showing increased WM relaxation with aging. The relaxation range in the WM (700-1000 ms, 50-30 ms; $\mathrm{T}_{1}, \mathrm{~T}_{2}$ ) was similar to our results. Korzdorfer et at [20] studied a 2D-MRF acquisition with seven slices and different field strengths (1.5 $\mathrm{T}$ and $3 \mathrm{~T}$ ) in selected brain structures, identifying a longer relaxation for the thalamus and caudate (1200-1400 ms, 40-50 ms; $\mathrm{T}_{1}, \mathrm{~T}_{2}$ ) compared to WM, also consistent with the findings of this investigation. The novelty of our study is the introduction of a whole-brain 3D-MRF acquisition that is able to describe similar results obtained from other studies but with an isotropic $1 \mathrm{~mm}^{3}$ resolution allowing the reconstruction of a 512 axial, coronal or sagittal slices. Therefore, the entire brain can be depicted. Importantly, this study was performed on a scanner from a different manufacturer with an acquisition time that is even shorter than those mentioned studies (acquisition length was oma with $\mathrm{T}_{1}$ and $\mathrm{T}_{2}$ relaxation times of $2020 \mathrm{~ms}$ and $158 \mathrm{~ms}$ respectively. c Axial post-gadolinium $\mathrm{T}_{1}$ WI shows the non-homogenous enhancement and higher internal cystic component with resultant longer relaxation times both on $\mathrm{T}_{1}$ and $\mathrm{T}_{2}$

between 5 [20] and $10 \mathrm{~min}$ [45]). The reduced acquisition time allows the proposed sequence to attain robust clinical feasibility and shows that MRF-based relaxation may be manufacturer agnostic.

The use of MRF-based $\mathrm{T}_{1}$ and $\mathrm{T}_{2}$ relaxometry represents an evolution of the concept of relaxometry as a biomarker of disease in the diagnosis and staging of intracranial masses [24, 42]. As early as 1980 s, investigators described using two different spin echo and one inversion recovery pulse sequences to compute relaxation values of meningioma [46, 47]. The study by Komiyama et al. [46] indicated that $T_{1}$ and $T_{2}$ values were greater for meningioma compared to anatomical structures due to higher water content, a finding consistent with the results reported in this work. Similarly, MRF-based relaxometry values for intra-parenchymal brain tumors have been reported to be greater than normal tissue 


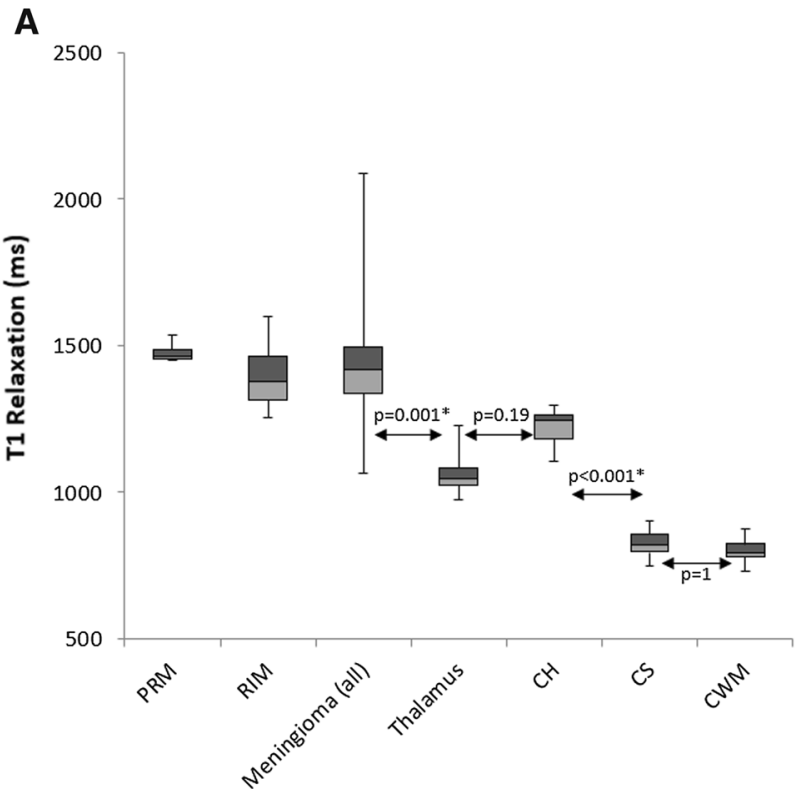

Fig. 5 Box and whisker plots for differences in $T_{1}$ (a) and $T_{2}(\mathbf{b})$ relaxation times between meningiomas and normal brain structures. The longitudinal lines depict the ranges, the light gray box the second quartile, the dark gray the third quartile and the solid horizontal line the median. For the meningiomas, the 25th percentile $\left(\mathrm{T}_{1}\right.$; $\left.\mathrm{T}_{2}: 1335 \mathrm{~ms} ; 51 \mathrm{~ms}\right)$, median $\left(\mathrm{T}_{1} ; \mathrm{T}_{2}: 1416 \mathrm{~ms} ; 65 \mathrm{~ms}\right), 75$ percentile
B

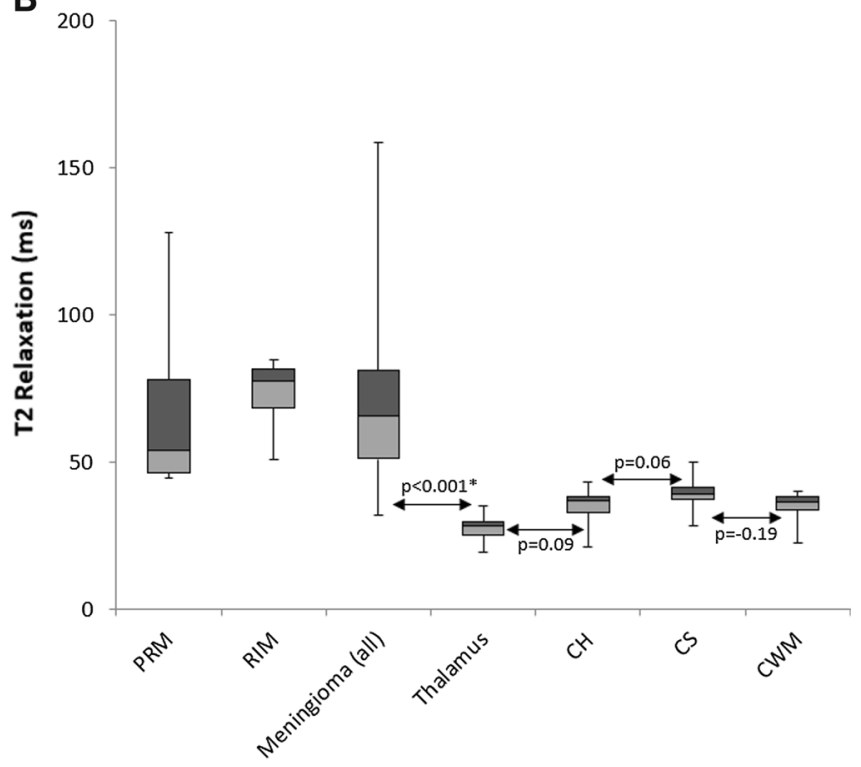

$\left(\mathrm{T}_{1} ; \mathrm{T}_{2}: 1493 \mathrm{~ms} ; 81 \mathrm{~ms}\right)$ were higher compared to all the anatomical structures. The different groups of meningiomas showed similar $\mathrm{T}_{1}$ and $\mathrm{T}_{2}$ relaxation times. $P R M$ partially resected meningioma, $R I M$ radiation induced meningioma, $\mathrm{CH}$ caudate head, $\mathrm{CS}$ centrum semiovale, $C W M$ contralateral white matter. ${ }^{*} p<0.05$ connotes statistical significance
Table $1 \mathrm{~T}_{1}$ and $\mathrm{T}_{2}$ MRF-based relaxometry values for all patients

\begin{tabular}{rllllll}
\hline & $\mathrm{T}_{1}$ mean $(\mathrm{ms})$ & $\mathrm{SD} \mathrm{T}_{1}{ }^{1}(\mathrm{~ms})$ & $p$ & $\mathrm{~T}_{2}$ mean $(\mathrm{ms})$ & $\mathrm{SD} \mathrm{T}_{2}{ }^{1}(\mathrm{~ms})$ & $p$ \\
\hline Meningioma $(n=25)$ & 1429 & 202 & Reference & 69 & 27 & Reference \\
Thalamus $(n=20)$ & 1054 & 58 & $\mathbf{0 . 0 0 1}^{*}$ & 27 & 3 & $<\mathbf{0 . 0 0 1 *}$ \\
$\mathrm{CH}(n=20)$ & 1223 & 52 & 0.19 & 35 & 5 & $<\mathbf{0 . 0 0 1} *$ \\
$\mathrm{CS}(n=20)$ & 825 & 42 & $<\mathbf{0 . 0 0 1} *$ & 29 & 5 & 0.15 \\
$\mathrm{CWM}(n=20)$ & 799 & 45 & $<\mathbf{0 . 0 0 1} *$ & 35 & 4 & $<\mathbf{0 . 0 0 1} *$
\end{tabular}

CS centrum semiovale, $\mathrm{CH}$ caudate head, $\mathrm{CWM}$ contralateral white matter

${ }^{*} p<0.05$ connotes statistical significance; values on bold are considered statistically significant

${ }^{\mathrm{a}}$ Standard deviation along the mean $\mathrm{T}_{1}$ and $\mathrm{T}_{2}$ among patients
[23]. However, the advantage of MRF-based estimates is reduced acquisition time when compared to conventional $\mathrm{T}_{1}$ and $\mathrm{T}_{2}$ mapping techniques, simultaneous multi-parameter relaxometry mapping, improved accuracy, repeatability and reproducibility based on phantom experiments [15] and invivo studies [20]. It is therefore feasible that MRF could be used as a clinical tool to rapidly and accurately quantify relaxation times as a biomarker of disease for meningiomas as well as other intracranial brain masses [23, 24, 40].

Quantitative MRI acquisitions other than MRF have been proposed to further elucidate the histological features of meningiomas, such as MR elastography and its basedstiffness estimates [34] as a possible presurgical planning tool [48]. In addition, ADC values acquired from DWI have been shown to correlate with aggressiveness of meningioma with lower ADC values indicating more aggressive meningioma [31, 32]. Recently, Zhang et al. correlated longer MRF-based relaxations with softer meningioma consistency after surgical resection, although the stiffness evaluation in this work was subjective [49]. In this case, MRF-based relaxometry depicts quantitative information of meningioma stiffness, but importantly it may be capable of differentiating meningioma based on grade. Peritumoral edema is a feature that may indicate more invasive meningioma [50], which in turn could led to a longer relaxation as analogous to previously published MRF-based correlation of increasing brain glioma tumor grade with prolongation of relaxation times [23, 24]. However, the use of MRF-based relaxation for predicting meningioma grade needs a much larger number of comparisons to design a statistically meaningful analysis 
than performed in this investigation, and given only three meningiomas were Grade II, we were not able to perform any statistical test for testing differences between Grade I and Grade II tumors. Importantly, surgical and treatment planning of meningioma are influenced by their histological grade $[30-32,48,51]$ and if MRF could distinguish this, it could have major clinical applications.

There are several limitations of this study. The sample size was small and was not powered to detect differences between different types of meningioma. While statistical significance was detected between meningioma and brain structures, a larger sample size would likely provide a powered study to further evaluate these differences. Second, the diagnosis of meningioma was based on imaging findings and only nine lesions had histologic confirmation. Ideally, complete classification of meningioma would require histological analysis, radiation status and tumor growth so as-to more accurately correlate with MRF-based relaxometry values.

\section{Conclusions}

Whole-brain 3D-MR fingerprinting relaxometry estimates have strong linear relationship with nominal values under experimental phantom studies for the expected in-vivo brain relaxometry values. Importantly, the short acquisition allows MR fingerprinting to be feasible in a clinical setting where relaxometry properties in meningiomas can be characterized using a high spatial resolution of $1 \mathrm{~mm}^{3}$ and potentially shortening MRI time when surveillance of those tumors is warranted. Although this analysis was not empowered enough to assess the potential role of MRF-based relaxometry as biomarker of tumor grade, the promising features we described with MR fingerprinting may guide investigations with larger samples.

Author contributions Conceptualization: TRM; AP; KPM; RJW; NGC. Methodology: TRM; AP; KPM; RJW. Formal analysis and investigation: TRM; AP. Writing — original draft preparation: TRM; KPM. Writing—review and editing: KPM; RJW; NGC; AL; YS.

Funding This research was institutionally funded by the Center of Individualized Medicine at the Mayo Clinic in Rochester, MN.

\section{Declarations}

Ethical approval This research was approved by the Mayo Clinic Institutional review board and all patients were consented.

Conflict of Interest The authors have no conflict of interest to disclose.

Open Access This article is licensed under a Creative Commons Attribution 4.0 International License, which permits use, sharing, adaptation, distribution and reproduction in any medium or format, as long as you give appropriate credit to the original author(s) and the source, provide a link to the Creative Commons licence, and indicate if changes were made. The images or other third party material in this article are included in the article's Creative Commons licence, unless indicated otherwise in a credit line to the material. If material is not included in the article's Creative Commons licence and your intended use is not permitted by statutory regulation or exceeds the permitted use, you will need to obtain permission directly from the copyright holder. To view a copy of this licence, visit http://creativecommons. org/licenses/by/4.0/.

\section{References}

1. Panda A, Mehta BB, Coppo S, Jiang Y, Ma D, Seiberlich N, Griswold MA, Gulani V (2017) Magnetic resonance fingerprintingan overview. Curr Opin Biomed Eng 3:56-66. https://doi.org/10. 1016/j.cobme.2017.11.001

2. Jiang Y, Ma D, Seiberlich N, Gulani V, Griswold MA (2015) MR fingerprinting using fast imaging with steady state precession (FISP) with spiral readout. Magn Reson Med 74(6):1621-1631. https://doi.org/10.1002/mrm.25559

3. Ma D, Gulani V, Seiberlich N, Liu K, Sunshine JL, Duerk JL, Griswold MA (2013) Magnetic resonance fingerprinting. Nature 495(7440):187-192. https://doi.org/10.1038/nature11971

4. Gómez PA, Molina-Romero M, Buonincontri G, Menzel MI, Menze BH (2019) Designing contrasts for rapid, simultaneous parameter quantification and flow visualization with quantitative transient-state imaging. Sci Rep 9(1):8468. https://doi.org/10. 1038/s41598-019-44832-w

5. Kurzawski JW, Cencini M, Peretti L, Gómez PA, Schulte RF, Donatelli G, Cosottini M, Cecchi P, Costagli M, Retico A, Tosetti M, Buonincontri G (2020) Retrospective rigid motion correction of three-dimensional magnetic resonance fingerprinting of the human brain. Magn Reson Med 84(5):2606-2615. https://doi. org/10.1002/mrm.28301

6. Ma D, Jiang Y, Chen Y, McGivney D, Mehta B, Gulani V, Griswold M (2018) Fast 3D magnetic resonance fingerprinting for a whole-brain coverage. Magn Reson Med 79(4):2190-2197. https://doi.org/10.1002/mrm.26886

7. Ma D, Jones SE, Deshmane A, Sakaie K, Pierre EY, Larvie M, McGivney D, Blümcke I, Krishnan B, Lowe M, Gulani V, Najm I, Griswold MA, Wang ZI (2019) Development of high-resolution 3D MR fingerprinting for detection and characterization of epileptic lesions. J Magn Reson Imaging 49(5):1333-1346. https:// doi.org/10.1002/jmri.26319

8. Ma D, Pierre EY, Jiang Y, Schluchter MD, Setsompop K, Gulani V, Griswold MA (2016) Music-based magnetic resonance fingerprinting to improve patient comfort during MRI examinations. Magn Reson Med 75(6):2303-2314. https://doi.org/10.1002/mrm. 25818

9. Cao X, Ye H, Liao C, Li Q, He H, Zhong J (2019) Fast 3D brain MR fingerprinting based on multi-axis spiral projection trajectory. Magn Reson Med 82(1):289-301. https://doi.org/10.1002/mrm. 27726

10. Asslander J (2020) A Perspective on MR Fingerprinting. J Magn Reson Imaging. https://doi.org/10.1002/jmri.27134

11. Poorman ME, Martin MN, Ma D, McGivney DF, Gulani V, Griswold MA, Keenan KE (2020) Magnetic resonance fingerprinting Part 1: Potential uses, current challenges, and recommendations. J Magn Reson Imaging 51(3):675-692. https://doi.org/10.1002/ jmri.26836 
12. Panda A, Chen Y, Ropella-Panagis K, Ghodasara S, Stopchinski M, Seyfried N, Wright K, Seiberlich N, Griswold M, Gulani V (2019) Repeatability and reproducibility of 3D MR fingerprinting relaxometry measurements in normal breast tissue. J Magn Reson Imaging 50(4):1133-1143. https://doi.org/10.1002/jmri.26717

13. Panda A, O'Connor G, Lo WC, Jiang Y, Margevicius S, Schluchter M, Ponsky LE, Gulani V (2019) Targeted biopsy validation of peripheral zone prostate cancer characterization with magnetic resonance fingerprinting and diffusion mapping. Invest Radiol 54(8):485-493. https://doi.org/10.1097/RLI.0000000000000569

14. McGivney DF, Boyacioglu R, Jiang Y, Poorman ME, Seiberlich N, Gulani V, Keenan KE, Griswold MA, Ma D (2020) Magnetic resonance fingerprinting review part 2: technique and directions. J Magn Reson Imaging 51(4):993-1007. https://doi.org/10.1002/ jmri.26877

15. Jiang Y, Ma D, Keenan KE, Stupic KF, Gulani V, Griswold MA (2017) Repeatability of magnetic resonance fingerprinting T1 and T2 estimates assessed using the ISMRM/NIST MRI system phantom. Magn Reson Med 78(4):1452-1457. https://doi.org/10.1002/ mrm.26509

16. Amthor T, Doneva M, Koken P, Sommer K, Meineke J, Bornert P (2017) Magnetic resonance fingerprinting with short relaxation intervals. Magn Reson Imaging 41:22-28. https://doi.org/10. 1016/j.mri.2017.06.014

17. Chen Y, Panda A, Pahwa S, Hamilton JI, Dastmalchian S, McGivney DF, Ma D, Batesole J, Seiberlich N, Griswold MA, Plecha D, Gulani V (2019) Three-dimensional MR fingerprinting for quantitative breast imaging. Radiology 290(1):33-40. https:// doi.org/10.1148/radiol.2018180836

18. Panda A, Obmann VC, Lo WC, Margevicius S, Jiang Y, Schluchter M, Patel IJ, Nakamoto D, Badve C, Griswold MA, Jaeger I, Ponsky LE, Gulani V (2019) MR fingerprinting and ADC mapping for characterization of lesions in the transition zone of the prostate gland. Radiology 292(3):685-694. https://doi.org/10.1148/radiol. 2019181705

19. Cruz G, Jaubert O, Qi H, Bustin A, Milotta G, Schneider T, Koken P, Doneva M, Botnar RM, Prieto C (2020) 3D free-breathing cardiac magnetic resonance fingerprinting. NMR Biomed 33(10):e4370. https://doi.org/10.1002/nbm.4370

20. Korzdorfer G, Kirsch R, Liu K, Pfeuffer J, Hensel B, Jiang Y, Ma D, Gratz M, Bar P, Bogner W, Springer E, Lima Cardoso P, Umutlu L, Trattnig S, Griswold M, Gulani V, Nittka M (2019) Reproducibility and repeatability of MR fingerprinting relaxometry in the human brain. Radiology 292(2):429-437. https://doi. org/10.1148/radiol.2019182360

21. Buonincontri G, Biagi L, Retico A, Cecchi P, Cosottini M, Gallagher FA, Gomez PA, Graves MJ, McLean MA, Riemer F, Schulte RF, Tosetti M, Zaccagna F, Kaggie JD (2019) Multi-site repeatability and reproducibility of MR fingerprinting of the healthy brain at 1.5 and 3.0T. Neuroimage 195:362-372. https:// doi.org/10.1016/j.neuroimage.2019.03.047

22. Wang K, Cao X, Wu D, Liao C, Zhang J, Ji C, Zhong J, He H, Chen Y (2019) Magnetic resonance fingerprinting of temporal lobe white matter in mesial temporal lobe epilepsy. Ann Clin Transl Neurol 6(9):1639-1646. https://doi.org/10.1002/acn3. 50851

23. Badve C, Yu A, Dastmalchian S, Rogers M, Ma D, Jiang Y, Margevicius S, Pahwa S, Lu Z, Schluchter M, Sunshine J, Griswold M, Sloan A, Gulani V (2017) MR fingerprinting of adult brain tumors: initial experience. AJNR Am J Neuroradiol 38(3):492499. https://doi.org/10.3174/ajnr.A5035

24. de Blank P, Badve C, Gold DR, Stearns D, Sunshine J, Dastmalchian S, Tomei K, Sloan AE, Barnholtz-Sloan JS, Lane A, Griswold M, Gulani V, Ma D (2019) Magnetic resonance fingerprinting to characterize childhood and young adult brain tumors.
Pediatr Neurosurg 54(5):310-318. https://doi.org/10.1159/00050 1696

25. Lu L, Chen Y, Shen C, Lian J, Das S, Marks L, Lin W, Zhu T (2020) Initial assessment of 3D magnetic resonance fingerprinting (MRF) towards quantitative brain imaging for radiation therapy. Med Phys 47(3):1199-1214. https://doi.org/10.1002/mp.13967

26. Marty B, Lopez Kolkovsky AL, Araujo ECA, Reyngoudt H (2020) Quantitative skeletal muscle imaging using 3D MR fingerprinting with water and fat separation. J Magn Reson Imaging. https://doi. org/10.1002/jmri.27381

27. Chen Y, Fang Z, Hung SC, Chang WT, Shen D, Lin W (2020) High-resolution 3D MR Fingerprinting using parallel imaging and deep learning. Neuroimage 206:116329. https://doi.org/10.1016/j. neuroimage.2019.116329

28. Keil VC, Bakoeva SP, Jurcoane A, Doneva M, Amthor T, Koken P, Madler B, Luchters G, Block W, Wullner U, Hattingen E (2020) A pilot study of magnetic resonance fingerprinting in Parkinson's disease. NMR Biomed 33(11):e4389. https://doi.org/10.1002/ nbm. 4389

29. Lamszus K (2004) Meningioma pathology, genetics, and biology. J Neuropathol Exp Neurol 63(4):275-286. https://doi.org/10.1093/ jnen/63.4.275

30. Marosi C, Hassler M, Roessler K, Reni M, Sant M, Mazza E, Vecht C (2008) Meningioma. Crit Rev Oncol Hematol 67(2):153171. https://doi.org/10.1016/j.critrevonc.2008.01.010

31. Filippi CG, Edgar MA, Ulug AM, Prowda JC, Heier LA, Zimmerman RD (2001) Appearance of meningiomas on diffusionweighted images: correlating diffusion constants with histopathologic findings. AJNR Am J Neuroradiol 22(1):65-72

32. Toh CH, Castillo M, Wong AM, Wei KC, Wong HF, Ng SH, Wan YL (2008) Differentiation between classic and atypical meningiomas with use of diffusion tensor imaging. AJNR Am J Neuroradiol 29(9):1630-1635. https://doi.org/10.3174/ajnr. A1170

33. Piper RJ, Mikhael S, Wardlaw JM, Laidlaw DH, Whittle IR, Bastin ME (2016) Imaging signatures of meningioma and low-grade glioma: a diffusion tensor, magnetization transfer and quantitative longitudinal relaxation time MRI study. Magn Reson Imaging 34(4):596-602. https://doi.org/10.1016/j.mri.2015.12.006

34. Murphy MC, Huston J 3rd, Glaser KJ, Manduca A, Meyer FB, Lanzino G, Morris JM, Felmlee JP, Ehman RL (2013) Preoperative assessment of meningioma stiffness using magnetic resonance elastography. J Neurosurg 118(3):643-648. https://doi.org/10. 3171/2012.9.Jns 12519

35. Gomez PA, Cencini M, Golbabaee M, Schulte RF, Pirkl C, Horvath I, Fallo G, Peretti L, Tosetti M, Menze BH, Buonincontri G (2020) Rapid three-dimensional multiparametric MRI with quantitative transient-state imaging. Sci Rep 10(1):13769. https://doi. org/10.1038/s41598-020-70789-2

36. Buonincontri G, Sawiak SJ (2016) MR fingerprinting with simultaneous B1 estimation. Magn Reson Med 76(4):1127-1135. https://doi.org/10.1002/mrm.26009

37. Cruz G, Schneider T, Bruijnen T, Gaspar AS, Botnar RM, Prieto C (2018) Accelerated magnetic resonance fingerprinting using softweighted key-hole (MRF-SOHO). PLoS ONE 13(8):e0201808. https://doi.org/10.1371/journal.pone.0201808

38. Russek SE, Boss M, Jackson EF, Jennings DL, Evelhoch JL, Gunter Jl, Sorensen AG (2012) Characterization of NIST/ISMRM MRRI system phantom. In: Paper presented at the ISMRM 20th Annual Meeting and Exhibition, Melbourne, Australia, May $5-11$ th

39. Yushkevich PA, Piven J, Hazlett HC, Smith RG, Ho S, Gee JC, Gerig G (2006) User-guided 3D active contour segmentation of anatomical structures: significantly improved efficiency and reliability. Neuroimage 31(3):1116-1128. https://doi.org/10.1016/j. neuroimage.2006.01.015 
40. Haubold J, Demircioglu A, Gratz M, Glas M, Wrede K, Sure U, Antoch G, Keyvani K, Nittka M, Kannengiesser S, Gulani V, Griswold M, Herrmann K, Forsting M, Nensa F, Umutlu L (2020) Non-invasive tumor decoding and phenotyping of cerebral gliomas utilizing multiparametric (18)F-FET PET-MRI and MR fingerprinting. Eur J Nucl Med Mol Imaging 47(6):1435-1445. https://doi.org/10.1007/s00259-019-04602-2

41. Liao C, Wang K, Cao X, Li Y, Wu D, Ye H, Ding Q, He H, Zhong J (2018) Detection of lesions in mesial temporal lobe epilepsy by using mr fingerprinting. Radiology 288(3):804-812. https://doi. org/10.1148/radiol.2018172131

42. Badve C, Yu A, Rogers M, Ma D, Liu Y, Schluchter M, Sunshine J, Griswold M, Gulani V (2015) Simultaneous T(1) and T(2) brain relaxometry in asymptomatic volunteers using magnetic resonance fingerprinting. Tomography 1(2):136-144. https://doi. org/10.18383/j.tom.2015.00166

43. Spijkerman JM, Petersen ET, Hendrikse J, Luijten P, Zwanenburg JJM (2018) T (2) mapping of cerebrospinal fluid: 3T versus 7 T. MAGMA 31(3):415-424. https://doi.org/10.1007/ s10334-017-0659-3

44. Kobayashi Y, Terada Y (2019) Diffusion-weighting caused by spoiler gradients in the fast imaging with steady-state precession sequence may lead to inaccurate T(2) measurements in MR fingerprinting. Magn Reson Med Sci 18(1):96-104. https://doi.org/ 10.2463/mrms.tn.2018-0027

45. Badve C, Yu A, Rogers M, Ma D, Liu Y, Schluchter M, Sunshine J, Griswold M, Gulani V (2015) Simultaneous T1 and T2 brain relaxometry in asymptomatic volunteers using magnetic resonance fingerprinting. Tomography 1(2):136-144. https://doi.org/ 10.18383/j.tom.2015.00166

46. Komiyama M, Yagura H, Baba M, Yasui T, Hakuba A, Nishimura S, Inoue Y (1987) MR imaging: possibility of tissue characterization of brain tumors using $\mathrm{T} 1$ and $\mathrm{T} 2$ values. AJNR Am J Neuroradiol 8(1):65-70

47. Watabe T, Azuma T (1989) T1 and T2 measurements of meningiomas and neuromas before and after Gd-DTPA. AJNR Am J Neuroradiol 10(3):463-470

48. Watanabe K, Kakeda S, Yamamoto J, Ide S, Ohnari N, Nishizawa S, Korogi Y (2016) Prediction of hard meningiomas: quantitative evaluation based on the magnetic resonance signal intensity. Acta Radiol 57(3):333-340. https://doi.org/10.1177/0284185115 578323

49. Zhang R, Bai Y, Zhang X, Feng Q, Wang M, Zhang M, Nittka M, Koerzdoerfer G, Lin Y, Wang M (2020) Magnetic resonance fingerprinting for preoperative differentiation of soft and hard meningiomas. In: Paper presented at the ISMRM \& SMRT Virtual Conference \& Exhibition, Virtual Meeting, August 10th, 2020August 14th, 2020

50. Kim BW, Kim MS, Kim SW, Chang CH, Kim OL (2011) Peritumoral brain edema in meningiomas: correlation of radiologic and pathologic features. J Korean Neurosurg Soc 49(1):26-30. https:// doi.org/10.3340/jkns.2011.49.1.26

51. Tamrazi B, Shiroishi MS, Liu CS (2016) Advanced imaging of intracranial meningiomas. Neurosurg Clin N Am 27(2):137-143. https://doi.org/10.1016/j.nec.2015.11.004

Publisher's Note Springer Nature remains neutral with regard to jurisdictional claims in published maps and institutional affiliations. 